\title{
Berliner Dissertationen
}

Willy Knoll-Berlin, Ein Beitrag zur Geschichte der Lepra.

German- G-adjatsch, Beitrag zur Kenntnis der Gehirnsyphilis ir $\alpha$ Kindesalter.

Held-Bromborg, Ein Fall von persistierender Erythrodermia idio-pathica.

Hermann En go Is-Berlin, Ueber Prurigo.

Fel sell -Hersfeld, Purpura rheumatica nach Mittelohreiterung.

Kudicke-Pr. Eylau, Uebor den Zusammenhang zwischen Asthma und Hautkrankhoiten.

Carl Kurtz-Berlin, Die fixon Antipyrinexantheme.

8. $\Lambda$ Vilhelm S chulze-Naumburg, Serumexantheine bei Diphtherie.

i). Lowek-Santomischel, Albuminurie bei Gonorrhoe.

II e c k z e h - Berlin, Tabes dorsalis und Niorenaffektionen.

Braunschmidt-Neustadt, Tabes dorsalis mit spätsyphilitischen Krank-heitserscheinungen.

Ernst Kuhn-Berlin, Häufigkeit des Vorkommens von Lues in der Anamnese von Tabischen und Nichttabischen.

.13. Guttmann-Posen, Tabes dorsalis und Syphilis.

Die Knoll'sche Arbeit (1) bedeutet weniger einen Beitrag zur Geschiehte der Lepra, als sie uns eine Vorstellung davon giebt, wie unklar die Ansichten der alten Aerzte über die Lepra, ihre Aotiologie und Behand-lung $\Lambda$ varen. Die Abhandlung giebt die Ansichten wiedcr, die Henri de Mondeville, ein berühmter Chirurg aus dor Schule de St. Come zu Paris, der Lehror Guy de Chauliacs über den Ausatz in seiner ,grossen Chirurgie” niedergelegt hat (1306). Interessant ist es zu erfahren, dass am Ende des 13. Jahrhunderts in Frankreich 2000, in ganz Europa etwa 19000 Leproserien bestanden. (!) Wieviel andere Dermatosen, wieviel Lues mag da als Lepra gosegelt sein! Mondeville führt die Krankheit auf die Materie der schwarzen Galle zurück. Hire Unheilbarkeit erkennt er im grossen und ganzen an. Als Ursachen giebt er hereditäre Belastung an, andererseits lasso sich die Lepra auch übertragon (durch Geschlechtsverkehr mit Leprosen). Drittens entstehe sie durch Coitus während der Menses. Die Symptome des Leidens beschroibt Mondeville im allgemoinen richtig, ihre Erklärung ist wie die Therapie im grössten Teil ganz unsinnig. Therapoutisch empfiehlt er kalte , feuchte Diät, Abführmittel, Aderlass, Blutegel, Cauterisationen, Schwitzkuren, Bäder, Massage, Salben, Pilaster und Umschläge. Ein Beispiel mag genügen: Man lege lebendige Schlangen, Fenchel- und Düll-Knollen Thymian oder Engelsüss und Anis in Most oder Wein, lässt diesen gähren. Umgefüllt soil dies Getränk so lange mit Wasser genossen werden, bis der Kranke Linderung verspürt. Sehr moralisch ist auch folgender Rat: ,,Hat Jemand mit einer Leprösen coitiert und fühlt ein Brennen am Penis, so soli er, bevor er noch Urin geiassen hat oder gleich darauf, mit einer gesunden Frau coitioren; dann wird diese infiziert sein".

German (2) giebt eine recht verdienstvolle Zusammonstollung der Fälle von Hirnlues, die bisher bei Kindern beobachtet worden sind. Wie die Hebung der Kenntnisse von der Nervensyphilis erst aus den letzten 50 Jahren datiert, so erst recht die Erfahrungen über die Hirnsyphiiis. John Hunter und Astley Cooper, die das Gehirn fur unempfänglich für Syphilis hielten, hinderten die Entwicklung auf diesem Gebiete. Die erste erschöpfonde Darstellung über die Syphilis des 
Nervensystems gab Lange au der Jüngere (1860). Trotzdem blieb die Gehirnlues wenig berücksichtigt, und Henoch konnte trotz seines reichen Materials keinen Fall von zweifel-loser Hirnsyphiiis beobachten. Man muss sagen, dass diese Krankheit im Kindesalter wirklich selten auftritt, sehr selten, $\Lambda$ venn man nur das Sections-

$46^{*}$

694 Berliner Dissertationen.

resultat als Beweis gelten lässt. Wenn man aber anamnestisck, klinische und th $\beta$ rapeutische Momente zusammenfasst, nicht in diesem extremen Masse selten.

Man muss unterscheiden zwischen Störungen in der Funktion und anatomisclien Erkrankungen; letztere zerfallen in solclie, die durch Con-gestionen entstanden, keinen specifischen Charakter aufweisen und als para-syphilitisch im Sinne Fourni«r's gelten müssen, und solclie mit specifischen Symptomen, die auf wahren syphilitischen Veränderungen beruhen. Man muss sich auch hüten, für zufälîig auítretende Gehirnerkrankungen immer die bestehende Lues verantwortlich zu machen. Selbst der Erfolg antisyphilitischer Behandlung sei nicht beweisend (! Der Verfasser führt hier 2 Fälle an, die Baginsky für tuberculös erklärte - gleichzeitig bestehende Lungenaffection und Fehlen aller specifischen anamnestischen Daten - und mit Jodkali heilte! Der Keferent).

An der Hand eines Falles von Gummenbildung in der Arachnoidea eines Bmonatlichen Kindes stellt German die bisher beobachteten ein-schlägigen Fälle zusammen: Meningitis specifica 5 Fälle, solitäre Gummata des Gehirns $\delta$ Fälle, Endarteriitis cerebralis mit consecutiver Erweichung und Sclerose 13 Fälle.

Max Held (3) führt uns den vor 3 Jahren der Berliner Der-matologischen Gesellschaft vorgestellten Fall $\tau$ on persistierender, idio-pathischer Erythrodermie von Blaschko in seiner Abhandlung nochmals vor. Es handelt sich um eine 54jährige Frau, die seit $\delta$ Jahren in Blaschko's Behandlung stehend, seit 6 Jahren an einer hartnäckigen Hautkrankheit leidet. An beiden Oberarmen fi'mfmarkstück- bis handteller-grosse, erythematöse Plaques; linker Unterarm und Handrücken stark gerötet. Ellbeuge und einzelne Inselchen sind normal. Im allgemeinen handelt es sich um eine dunklere Färbung als am übrigen Kvrper. Es finden sich diffuse Bräunung, diffuse rotbraune Verfärbung, circumscripte, intensiver ge-rötete Plaques und tief dunkolblaurote Stellen nebeneinander. Verfasser geht die Differentialdiagnosen Erythema multiforme, nodosum, elevat'um diutinum, Congelatio durch, die er alle von der Hand weist. Nicht auf den ersten Blick auszuschliessen sind beginnende Scler o dermie, bei der Induration, Yerdickung und Spannungsgefühl in Bouttier's Fall erst nach $\bullet 1$ Jahren auftrat, beginnende Mycosis fun guides im Stadium der soge-nannten Erythrodermia, wie Louis Wickham einen Fall beschrieben hat und wie Kaposi einen vom Keferenten selbst gesehenen Fall der Wiener dermatologischen Gesellschaft präsentierte, Erythromelalgie, Erythro-melie (Pick) und Erythema perstans (Payne). Es bleibt abzuwarten, ob Scleroderma oder Mycosis iüngoides sich allmählich noch herausbilden, vorläufig halt Held das Leiden mit Blaschko für eine selbständige Dermatose, ein idiopathisches, chronisches Erythem, das viele Jahre zur Evolution gebraucht. Eine zusammenfassende Darstellung der Prurigo-Hebra giebt Engels (4). Im Altertum war Prurigo als eigne Dermatose unbekannt, Willan nannte jede juckende Hautkrankheit so, erst Hebra definierte die Begriffe und unterschied nach Heftigkeit der Symptome eine mitis und eine ferox. îsie congenital entsteht sie stets im 1. Lebensjahr als Quaddeln, scli $\Lambda$ vindet temporär, ist aber unheilbar. Hebra stellte die ïheorie auf, an gewissen Hautstelleii bestehe ein Plus von Intercellularflüssigkeit, die die Hornschicht vor sich hertreibt (Knötchen) und die Nerven des Papillarkörpers reizt (Jucken). Auspitz stellte die Prurigo unter seine Idioneurosen der Haut, will 
sie für eine Sensibilitätsneurose erklären, die Knötchen bedeuteten eine Contractibilitätsneurose; Neisser diagnosticiert Prurigo auch bei Entstehen in späteren Jahren, halt sie für eine Hautneurose mit Sensibilitäts- und vaso-motorischen Störungen, die Lokalisation erklärt er mit dem Nerven- und Gefässreichtum der betreffenden Prädilektionsstellen. Engels vertritt den Standpunkt der Wiener Schule gegenüber der franzosischen, die Prurigo und Pruritus zusammen werfen will. Er betont auch das hereditäre Moment, das schon Kaposi hervorhebt. Unter 140 Fallen aus der Joseph'schen Poliklinik sind 4 Geschwisterpaare. Besnier sagt, Prurigo käme nor bei Leuten mit abnormem Stoffwechsel vor (Prurigo diathésique). Die Ursache Berliner Dissertationen. 695 sei intern, die Yeranlassung extern (Scabies u. a.) Àls Bewëis führt er seine erfolgreiehe interne Medication an. Im allgemeinen steht Engels auf NOisser's aetiologischem Standpunkt. Im Gegensatz zujíaposi nehmen Joseph und cr auch eine Prurigo an, wenn das Leiden in späteren Jahren beginnt, $\lambda$ vo die Wiener Schule Eczema papulatum diagnosticíert. Joseph's poliklinisches Material weist 40,92 pCt. Prurigofälle auf. In England und Amerika soil die Krankheit selten sein, auf den Seychellen endemisch. Nebeu der Allgemeinbehandlung des Körpers, die roborierend sein muss, verwendet Joseph Naphtholsalben.

Die Arbeit Felschs $\varphi$ ) bietet wenig dermatologisches Interesse. An der Hand eines Falles von acutem Gclenkrheumatismus mit Purpura, der an eine acute Ohrenoiterung sich anschloss, bespricht der Verfasser die Aetio-logio der Polyarthritis. Die Frage, ob wir es hier mit eineni specitischen Microorganismus, den Koos, Fiedler u. a. annebmen, zu thun haben, ver-neint er. Das Leiden kann an Infectionen mancherlei Art aDSchliessen, sei es an eitrige Anginen, Harnröhren-, Bronchial-, und Darmeiterungen. Wir müssen es also als eine Art Secundärinfection ansehen, deren Erreger ver-schiedene sein können. Der acute Gelenkrheumatismus ist als eine ab-geschwächte Pyämie zu betrachten.

Von grösstem Interesse ist dagegen die Abhandlung Kudickes (6), der den Zusammenhang des Asthmas mit Ilautkrankheiten und vice versa eingehend erörtert. Die Grundlage des Asthmas ist sicher eine nervöse, wofiir das anfallsweise Auftreten, das plötzliche $\Lambda$ Viedernachlasse, $n$ der Erschein $\pi$ ngen und das völlige Wohlbefinden in der Zwischenzeit sprechen. Das Asthma kann auf eine bestehende Dermatose steigernd einwirken oder bei leichter Irritabilität der cutanen Gewebe wie bei Gicht, Diabetes, Anämie und chronischer Eczematose direkt Eruptionen neu hervorrufen. Auch kann eine nicht zu acut verlaufende Hautkrankheit, die mit Reizung der sensiblen jN"ervenendigungen einhergeht, eine Fluxion in der Bronchialschleimhaut bez. einen Krampf der Bronchialmuskeln herbeiführen. Ein durch Asthmaanfälle gesteigerfes liautleiden wirkt dann aber wieder auf das Asthma verschlimmernd ein. Die unmittelbare Ursache sind die Gefässerweiterer, die entweder durch sensible Fascrn oder im andern Fall durch Lungenfasern des N. vagus gereizt werden. Es handelt sich hierbei immer um echtes Asthma, das schon im ersten Jahrzehnt des Lebens entstanden ist. Bei später auftretendem spielen die Ilautkrankheiten keine derartige Kolie. Aechte Asthmatiker werden immer angeben, in frühester Kindheit an Hautausschlägen gelitten zu haben (meist Ecsemen). Verfasser berichtet einen typischen Fall bei einem ISjährigen Arbeiter, dor an Eczem und Asthma leidend bei jedem Anfall Urticaria bekommt, und wo man andererseits beim Auftreten der Quaddeln auch jedesmal Lungenerscheinungen constatieren kann.

Die Arbeit von Carl Kurtz (7) beschäftigt sich mit den fixen Anti-pyrinexanthemen, den Eruptions fixes par l'antipyrine der Franzosen Es ist bekannt, dass die Hautausschläge zwar verhältnismässig die ungefährlichsten, aber doch recht lãstige Neb en wir kun gen des Mittels darstellen. Der Form nach sind die Antipyrinexantheme meist Erytheme, Urticariaquaddeln oder 
selten Purpuratlecke. Auch sind pemphigoide Eruptionen und Haut $\delta$ deme beobachtet worden. Die Ausschlägo treten entweder diffus oder localisiert auf. Es sind eine Anzahl Fälle berichtet, bei denen das Antipyrinexanthem sich bei jedesmaligem Gebrauch des Medicaments die gleiclie Localisation aussucht, selbst wenn $<$ Tahre da-zwischen liegon. Das sind diese sogenannten fixen Antipyrinexantheme. Merkwürdigerweise können sie mit Fiebersteigerung vorlaufen. Prädilections-stellen sind Gesicht, obere Extremitäten und Genitalien, besonders Streck-seiten. Sehr oft Ausgang von den Kürperöffnungon aus. Schiíttelfrost, Schwindel, Mattigkeit können hinzukommen. Jucken unerträglich. Differen-tialdiagnose oft sehr schwer gegen Morbilli, Scarlatina, besonders aber Urticaria und Syphilis. Kurtz reiht den bisher bekannten 15 Fallen 2 weitere an.

Wie das Antipyrin, so besitzt auch das so segenreich wirkende Diphtherie-Heilserum schädliche Neben $\Lambda$ vírkungen, zu denen vor alien die Hautausschläge zu zählon sind. (8). Sie treten in 5 Formen als Urticaria, morbilloid, scarlatinoid, unbestimmten und iocalen Characters auf. Das Alter

696

Berliner Dissertationen.

der Patienten hat keinen Einfluss hierauf. Nach einzelnen Sorumnummern häuften sich die Exanthéme besonders stark. Auch von der Menge des Serums, das eingespritzt wurde, scheint der Ausschlag nicht abzuhängen. Die Dauer beträgt 1-8 Tage. Bei den spat nach der Injection auftretenden Exanthemen stellen sich meist höhere Temperaturen ein (bis 40,8). Das Fieber geht meist einen Tag früher zurück wie der Ausschlag, kann ihn aber auch überdauern. Ohne Fieber verliefen in der von Wilhelm Schuizo (8) beschriebenen Reihe 39 pCt. der Fälle. Weitere zu den fiebcrhaften Serumexanthemen tretende und auf Rechnung des Serums zu setzende Schädigungen sind: Albuminurie, Drüsenschwellungen und Gelenkaffectionen. Aetiologisch wurde die Carbolsäure im Serum verantwortlich gemacht, $\Lambda$ vohl mit Unrecht, denn Johannessen $\Lambda$ vies Exanthe'me auch bei carbolfreiom Serum nach. Uel $>$ er die $\Lambda$ vahren TJrsachen wissen wir noch nichts.

Lewek (9) stellt in seiner Arbeit fest, dass das Yorkommen von Albuminurie im Verlaufe der Gonorrhoe nicht gar so selten ist, ganz im Gegensatz zur wahren Nephritis. Hauptsächlich den Franzosen verdanken wir die ersten Untersuchungen auf diesem Gebiet. Nach Bell (1800) künnen die Nieren beim Tripper par une sympathie nerveuse erkranken und durch eine aufsteigende Entzüdung. Fournier leugnet eine Nephritis gonorrhoica» Die Nierenschmerzen führt - er auf Copaivabalsam oder auf beginnende Epididymitis zuriick. $\Lambda$ Vichtig sind die Untersuchungen Balzers und Souplets mit 429 Krankenbeobac.htungen. In 23 pCt. der Fälle war Albuminurie zu finden. Diese Autoren unterscheiden leichte und schwere Albuminurie; ferner trennen sie pathogenetisch a) A. nach Cystitis und TJreterenpyelonephritis, b) nach Cystitis mit undeutlichen Syniptomen, c) bei Epididymitis, d) mit Epididymitis und gleichzeitiger Pyelonephritis. Die Balsamica schliessen sie als Ursache aus. Die Toxine des Gonococcus verändern das Nierenepithel. Goldberg unterscheidet die ascendierende Pyelonephritis und die Nierenentzündung durch Allgemeininfektion. Finger macht für die Albuminurie allein den Harndrang verant $\Lambda$ vortlich. Infolge des dabei bestehenden Harnleiterkrampfs tritt Harnstauung in die Niere ein, doren secretorischer Druck relativ sinkt, was nach Runebergs Theorie zur Eiweiss-ausscheidung führt. Leweks Material umfasst 155 Krauke mit (5 wahren Albuminurien. Das dabei bestehende Fieber, infolgo andrer Kvrperleiden,. wie Arthritis, Angina, Cystitis u. a. macht er für die Eiweissausscheidung verantwortlich, also keineswegs Toxine des Gonococcus. 
Yier Arbeiten beschäftigen sich mit der Tabes. Reckzeh (10) äussert sich über deren Yerhältnis zu Nierenleiden folgendermassen: Tabiker zoigen nicht selten als Complication Nierenkrankheiten, die im ursächlichen Zusammenhang mit der Tabes stehen. Es sind $\Lambda$ Vandernieren, Hydro-nephrosen, ascendierende Nephritiden, hauptsächlich aber chronisch paren-chymatöse und interstitielle Nierenentzündungen. Die Syphilis spielt dabei durch das Bindeglied dor Arterienerkrankungen eine wichtige ätio-logische Rolle. Braunschmidt (11) berichtet über zwei Fälle von Tabes mit gleichzeitig bestehenden spätsyphilitischen Erscheinungen. Ebe^ifalls die FournierErb'sche Lehre von der ätiologischen Rolle, die die Lues bei der l-iückenmarksschwindsucht spielt, vertritt die Abhandlung Kuan's (12), der eine Reihe von 214 Tabischen und 600 Nichttabischen in Betracht zieht und dabei einen 5 mal so grossen Prozentsatz von Tabischen als Nichttabischen mit sicherer syphilitischer Anamnese fand. Ganz im Gegensatz zu Erb ver tritt L e y d e $n$ mit seiner Schule den Standpunkt, dass die Lues mit der Tabes nichts zu thun habe (13). Ein umfangreiches Material steht hinter dieser Ausicht. Nach Becrwald findet sich unter don Eingeborenen Ostafrikas mit 50 pCt. Syphilis von alien Krankheitsf alien keine Tabes; dasselbe gilt nach Uäubler für Südostafrika. Grimm fand auf Jeddo, wo die Lues 10 pCt. aller Krankheiten ausmacht, unter 60000 Kranken nur 5 Tabiker. Neftel unter den stark durch Lues verseuchten Kirgisen keine Tabes, Glück ebensowenig unter der bosnischen BevvTkerung. Die Leyden'sche Schule verwirft infolgedessen auch die antisyphilitische Behandlung der Tabes. H o pf-Dresden. 